3-1-2008

\title{
Subsiding Shells Around Shallow Cumulus Clouds
}

Thijs Heus

Cleveland State University, t.heus@csuohio.edu

Harmen J.J. Jonker

Delft University of Technology, h.j.j.jonker@tudelft.nl

Follow this and additional works at: https://engagedscholarship.csuohio.edu/sciphysics_facpub

Part of the Physics Commons

How does access to this work benefit you? Let us know!

Publisher's Statement

(C) Copyright 2008 AMS

\section{Repository Citation}

Heus, Thijs and Jonker, Harmen J.J., "Subsiding Shells Around Shallow Cumulus Clouds" (2008). Physics Faculty Publications. 229.

https://engagedscholarship.csuohio.edu/sciphysics_facpub/229

This Article is brought to you for free and open access by the Physics Department at EngagedScholarship@CSU. It has been accepted for inclusion in Physics Faculty Publications by an authorized administrator of

EngagedScholarship@CSU. For more information, please contact library.es@csuohio.edu. 


\title{
Subsiding Shells around Shallow Cumulus Clouds
}

\author{
ThiJs Heus AND Harm J. J. Jonker \\ Department of Multi-Scale Physics, Delft University of Technology, Delft, Netherlands
}

(Manuscript received 18 October 2006, in final form 5 June 2007)

\begin{abstract}
In this study large-eddy simulations (LES) are used to gain more knowledge on the shell of subsiding air that is frequently observed around cumulus clouds. First, a detailed comparison between observational and numerical results is presented to better validate LES as a tool for studies of microscale phenomena. It is found that horizontal cloud profiles of vertical velocity, humidity, and temperature are in good agreement with observations. They show features similar to the observations, including the presence of the shell of descending air around the cloud. Second, the availability of the complete 3D dataset in LES has been exploited to examine the role of lateral mixing in the exchange of cloud and environmental air. The origin of the subsiding shell is examined by analyzing the individual terms of the vertical momentum equation. Buoyancy is found to be the driving force for this shell, and it is counteracted by the pressure-gradient force. This shows that evaporative cooling at the cloud edge, induced by lateral mixing of cloudy and environmental air, is the responsible mechanism behind the descending shell. For all clouds, and especially the smaller ones, the negative mass flux generated by the subsiding shell is significant. This suggests an important role for lateral mixing throughout the entire cloud layer. The role of the shell in these processes is further explored and described in a conceptual three-layer model of the cloud.
\end{abstract}

\section{Introduction}

The properties of shallow cumulus clouds have long been a much-studied topic in the research of atmospheric boundary layers. One important ongoing issue is the interaction between cloud and environment, despite the fact that it has been an open topic for more than half a century. Stommel (1947) based his cloud model on the concept of a lateral entraining plume, but Squires (1958) argued that cloud-top mixing and resulting penetrative downdrafts were better able to predict the behavior of cumulus clouds. Asai and Kasahara (1967) emphasized the role of environmental subsidence in compensation of the in-cloud updrafts. Paluch (1979), and after her, for example, Betts (1982), Jensen et al. (1985), Reuter and Yau (1987a), and Jonas (1990, hereafter J90), showed conserved variable diagrams of observations indicating that in-cloud air originates from either the subcloud layer or regions at or above cloud top. On the other hand, Blyth et al. (1988) and Taylor and Baker (1991), for instance, saw lateral mixing as

Corresponding author address: Thijs Heus, Delft University of Technology, P.O. Box 5046, 2600 GA, Delft, Netherlands.

E-mail: t.heus@tudelft.nl dominating mechanism, modeling the cloud using a buoyancy sorting mechanism. Blyth (1993) pressed the significance of a recirculating crown topping the cloud to be the cause of an increase in source level in the highest part of the cloud. Currently, many operational parameterizations are based on a mass-flux approach, which is based on a laterally mixing cloud field (see, e.g., Siebesma and Cuijpers 1995).

Apart from observational data, large-eddy simulations (LES) are very useful for research in this field, as they provide sufficient statistics and full information on the three-dimensional flow. However, detailed comparisons between LES and observations remain desirable. Usually (e.g., Neggers et al. 2003; Siebesma et al. 2003; Stevens et al. 2001) only slab-averaged fields and derived quantities are compared with observations. This paper attempts to take the validation of LES a step further by comparing the simulations with the observation by Rodts et al. (2003, hereafter RDJ03). In that study, detailed lateral profiles of the main (thermo-) dynamic variables conditionally averaged over clouds and surroundings are taken. To obtain optimal comparison, the numerical results are processed in the same way as the observational data, enabling a direct and fair comparison. 
The main focus of this paper lies at the shell of subsiding air found at the edge of the cloud as reported by, for example, J90 and RDJ03. This shell is also visible in results of, for example, Austin et al. (1985) and Zhao and Austin (2005), although the possible role of the shell in cloud physics is not explicitly discussed in these papers. Recently Siebert et al. (2006) observed that in the subsiding shell turbulence is much stronger than in either the cloud core or in the environment. This effect is due to enhanced shear in the shell and might cause a more homogeneous mixing at cloud edge, thus altering droplet spectra.

From the point of view of cloud dynamics the significance of the shell lies in the fact that it is a part of the cloud cell transporting air downward (like penetrative downdrafts do), but is located at the edge of the cloud, and thus is closely related to lateral mixing. The mechanism behind the shell can be explained by both cloudtop mixing and lateral mixing. If the influence of lateral mixing is negligible within this shell, as Reuter and Yau (1987b) and J90 suggested, the shell has to be driven by mechanical forcing through the pressure-gradient force. RDJ03, on the other hand, advocated that lateral mixing over the cloud edge causes evaporative cooling. This in its turn results in negatively buoyant air and descending motion alongside the cloud. Unfortunately, because of the lack of sufficient observational data, neither study could be conclusive on this subject. Not only did obtaining statistically reliable results prove to be difficult, moreover some necessary information on the possible forces behind the shell, and in particular the pressure gradient, was unavailable. As demonstrated by, for example, Siebesma and Jonker (2000) on subjects similar to this problem, the controllable environment of LES can provide a well-suited tool to study this subsiding shell. It enables the possibility to extract complete three-dimensional fields of all variables and ensemble averaging over many statistically independent simulations can ensure reliable statistics.

Thus, this paper aims to find conclusive evidence for the governing mechanisms behind the descending shell. To this end, in section 3 observations and LES are compared to validate the use of LES on detailed cloud dynamics. Next, the individual terms of the vertical momentum equation are analyzed to find the mechanism driving the shell in section 4. Hereafter, the occurrence of the shell in other numerical cases is discussed, along with various properties of the shell (section 5), including the relevance of the shell to the dynamics of the cloud and the cloud layer. A connection will be made here with the off-center position of the cloud core as seen by Heymsfield et al. (1978) and the "humidity halos" that are found by, for example, Perry and Hobbs (1996), Lu et al. (2003), and Laird (2005), and adjoin clouds. These halos are shown to be caused by lateral mixing and yield an increase in humidity of the environmental air especially at the downshear side of the cloud. Since halos are placed in the conditionally stable environmental air, they tend to remain for a relatively long time. This may result in more favorable conditions for growth of subsequent clouds, as suggested by Telford and Wagner (1980) and Kuang and Bretherton (2006).

The discussion finally leads to the development of a simple conceptual model of the cloud, the cloud edge, and the environment in section 6. Within this model, the role of the shell in the interaction between cloud and surroundings can be isolated from other processes and can be explored in detail.

\section{Description of the large-eddy simulations}

\section{a. Case description}

All simulations were performed with the parallelized Dutch Atmospheric LES (DALES) model of which the details are described by Cuijpers and Duynkerke (1993). For reasons discussed below, two idealized cases are studied here. First is the case designed by Neggers et al. (2003) based on the Small Cumulus Microphysics Study (SCMS) observations. Second is the case by Siebesma et al. (2003) of the Barbados Oceanographic and Meteorological Experiment (BOMEX; Holland and Rasmusson 1973).

Neggers et al. (2003) modeled their numerical case on measurements done on 5 August 1995 above Florida (see Knight and Miller 1998; French et al. 1999 for details). This day was part of a period where nonprecipitating shallow cumuli developed every day, and large-scale forcings were constant and small compared with the surface fluxes. The case is designed to run from sunrise at 1200 UTC till sunset at 2400 UTC. Sensible and latent surface heat fluxes are sinusoidally shaped with a maximum of 100 and $300 \mathrm{~W} \mathrm{~m}^{-2}$, respectively, at 1800 UTC. This results in a Bowen ratio $r_{B}$ of 0.3 . Cloud base is located around $700 \mathrm{~m}$ and cloud top at $2200 \mathrm{~m}$. The system is subject to a mean wind of $(-4,4)$ $\mathrm{m} \mathrm{s}^{-1}$ related to a sea breeze and a mean shear of approximately $(0,0.7) \mathrm{m} \mathrm{s}^{-1} \mathrm{~km}^{-1}$. To follow the cloud field being advected by this wind, a Galilean transform of $\mathbf{U}_{T}=(-4,4) \mathrm{m} \mathrm{s}^{-1}$ was imposed. Simulations were carried out on a domain of $6.4 \mathrm{~km} \times 6.4 \mathrm{~km} \times 5.12 \mathrm{~km}$ with each grid box of a size of $\Delta x=\Delta y=50 \mathrm{~m}, \Delta z=$ $40 \mathrm{~m}$ and a time step of $\Delta t=0.5 \mathrm{~s}$. For this study, data between 1800 and 2200 UTC were used, coinciding with 
the times of measurement flight RF12 during the SCMS campaign. To enhance statistics five simulations were carried out. All simulations are identical save for a random perturbation of the initial temperature and humidity field. This creates statistically identical but independent runs (see Chlond and Wolkau 2000).

The BOMEX case is described by Siebesma et al. (2003). Sensible and latent surface heat fluxes amount to 8 and $150 \mathrm{~W} \mathrm{~m}^{-2}$, respectively (yielding $r_{B}=0.05$ ). Cloud base is located around $600 \mathrm{~m}$ and cloud top at $1700 \mathrm{~m}$, meaning that clouds are also somewhat smaller than in SCMS. The mean geostrophic wind is set to $[-10+(z / 555), 0] \mathrm{m} \mathrm{s}^{-1}$ resulting in a shear of approximately $1.8 \mathrm{~m} \mathrm{~s}^{-1} \mathrm{~km}^{-1}$. Again, a Galilean transform is performed [here $\mathbf{U}_{T}=(-8,0) \mathrm{m} \mathrm{s}^{-1}$ ] to follow the cloud field. Ten simulations with different random initialization are carried out on a domain of $6.4 \mathrm{~km} \times$ $6.4 \mathrm{~km} \times 3.2 \mathrm{~km}$, a grid box of $\Delta x=\Delta y=25 \mathrm{~m}, \Delta z=$ $20 \mathrm{~m}$, and a time step of $\Delta t=1 \mathrm{~s}$. From each simulation the first $3 \mathrm{~h}$ are discarded as spinup.

While the work by RDJ03 on the SCMS case allows for a detailed comparison with those observations, it is interesting to see how dynamics of SCMS clouds compare with simulations of marine boundary layer of BOMEX. Especially for processes driven by phase changes like evaporative cooling, the more humid environment and lower Bowen ratio of BOMEX could very well result in different dynamics.

Moreover, according to Heymsfield et al. (1978) and Perry and Hobbs (1996), the enhanced vertical shear in BOMEX is important for processes associated with lateral mixing, and may be so here as well. BOMEX has also the practical advantage of a lack of diurnal cycle. This makes longer runs possible and ensures a statistically identical cloud field over the entire run. This results in enhanced statistics and enables exploration of, for example, the shell as a function of cloud size.

\section{b. Method of postprocessing}

The study of RDJ03 was based on flights RF12, RF13, RF16, and RF17 of the SCMS database, held on 5, 6, 10, and 11 August 1995. Their analysis consisted of a conditional sampling of all transects through clouds larger than $L_{c}=500 \mathrm{~m}$, normalized to unit length. After a correction for observation height, averages were taken of observational quantities (the vertical velocity $w$, the liquid potential temperature $\theta_{l}$, and the total water content $q_{t}$ ) over all transects. In averaging, the average value of the environment before entering the cloud has been subtracted from the sample.

For comparison between LES and observations, the sampling procedure in LES is modeled closely to the method of RDJ03. Virtual flights are taken through the LES domain and points are sampled within a cloud or within one cloud length distance from both sides of the cloud. These flights are usually taken at a fixed height, typically $1000 \mathrm{~m}$ above the surface. The average value of the region before the cloud was subtracted from all the values, and the results are averaged over all clouds. A slight alteration of RDJ03 was needed to fit the method on the LES grid. First, flights through clouds were only taken along LES grid lines in west-east and north-south direction and vice versa. Second, only transects of exactly $400 \mathrm{~m}$ were taken into account to avoid the need of rebinning, since that could smooth out much of the signal. The slight reduction in size to $L_{c}=400 \mathrm{~m}$ (compared to the $500 \mathrm{~m}$ in RDJ03) ensured that enough clouds exist for reliable statistics. While in observations the pilot attempted to fly through the center of active clouds, the trajectories were rather wide, resulting in inclusion of other transects through clouds. This allows for good comparison with the simulations where transects could be either through the center of a 400-m-sized cloud or through the size of a much bigger cloud.

If on a certain line of measurement one of the environmental points happens to fall inside another cloud, this entire line is discarded from the analysis, to avoid pollution of the statistics. Data within one gridpoint distance of the cloud top or bottom are discarded, to avoid biases due to averaging over the vertical border of the cloud.

\section{Comparison with observations}

For the validation of the numerical work, the flythrough profiles of $w, q_{t}$, and $\theta_{l}$ are compared with RDJ03. In Fig. 1, these results are plotted. The left column shows LES simulations, in the middle column are the RDJ03 results of averaging over all flights, and the results of individual flight days in RDJ03 are shown in the right column. On top of the average profiles the rms deviations are plotted for the LES and the average RDJ03 results. These deviations do not represent the error in the mean but rather a measure for the variability between different clouds. The corresponding graphs in Fig. 1 appear to match as well as can be expected; the average numerical result lies well within the natural variation of the observations.

However, it should be noted that both the values of $q_{t}$ and $\theta_{l}$ as well as the variation around the mean profiles is slightly smaller in LES than in the averaged observations. Several explanations can be given for this: first, the simulations are based on the measurements on 

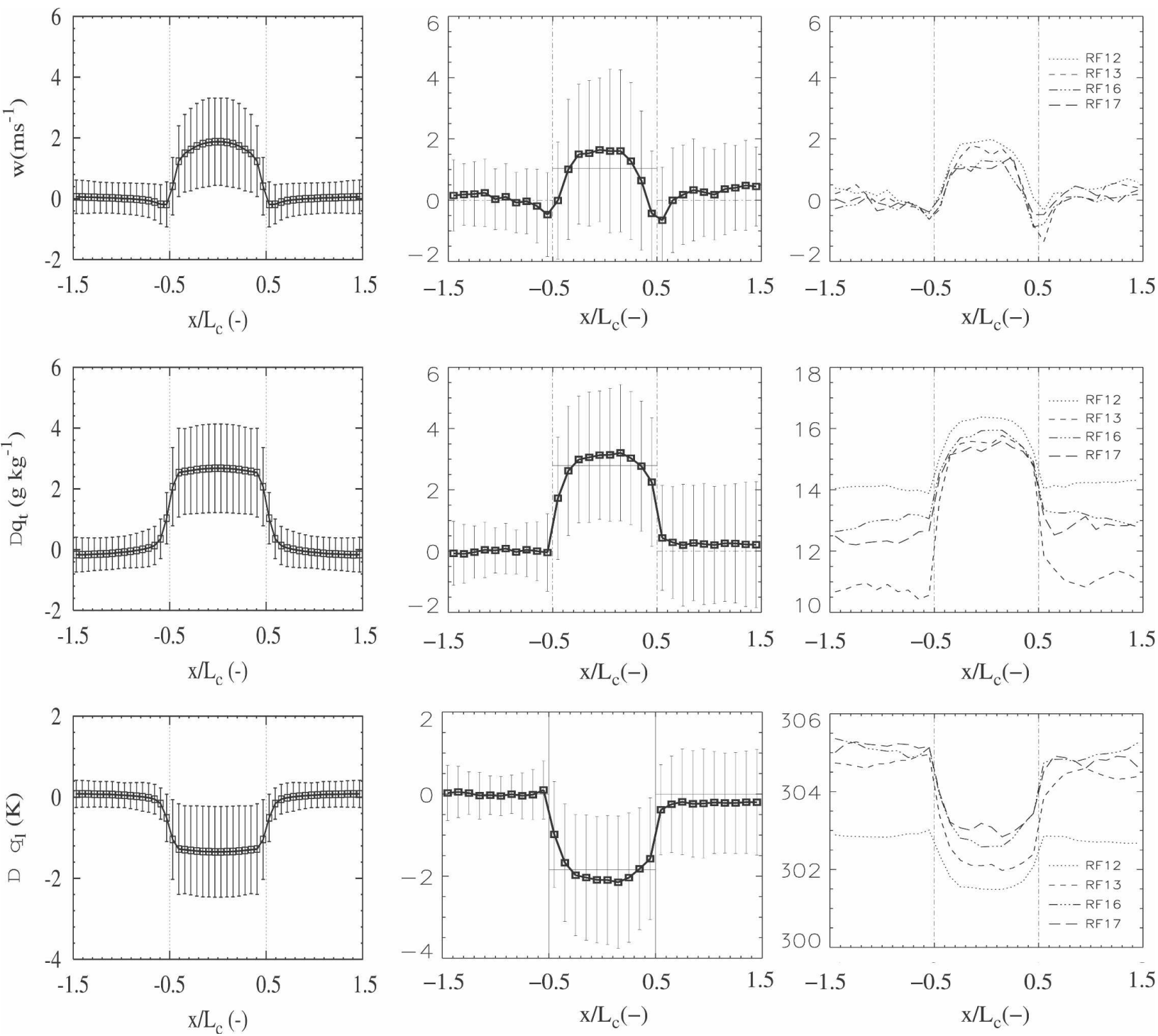

FIG. 1. Averaged in-cloud profiles of vertical velocity, total water content, and liquid water potential temperature of (left) the LES, (middle) the average over all flights by RDJ03, and (right) the individual flights by RDJ03. For the left and middle columns, the mean value left of the cloud is subtracted. The cloud is centered at zero and scaled with cloud size $L_{c}$. The bars denote the rms values of the individual measurements; these bars thus do not denote an error, but are a measure of variance between the various clouds. Horizontal lines in the middle column denote the environmental and in-cloud averages. For the observations, clouds of at least $500 \mathrm{~m}$ are taken into account; in the simulations, transects are taken at 1000-m height and only 400-m-sized clouds are presented.

5 August, the day of flight RF12, represented in 1 (right) by the dotted line. This flight resulted in belowaverage differences in liquid water potential temperature and total humidity. The fact that LES did not sample over the varying conditions of the various flight days also explains the smaller fluctuations. Second, since in LES only clouds of one fixed size are taken into account, it can be expected that the variation between different transects is also less.

The size of the subsiding shell, while still visible in the $w$ profile in LES, is less pronounced than in observa- tions. This can be attributed to the filtering procedure that underpins the LES methodology. An LES gridbox value represents an average over the size of the grid box $(25 \mathrm{~m})$, while observations were done with a spatial sampling rate of $(10 \mathrm{~m})^{-1}$. It is therefore likely that LES smoothes out more of the shell than observations do. Finally, in observations an asymmetry can be seen that is not visible in LES, because of the fact that in LES transects are taken from all sides. The origin of such asymmetries is discussed in section 5c. Overall, the most striking features of the observations, such as the 
cloud edge minimum in the $w$ profile, are clearly present and similarly sized in the simulations. LES thus seems to be a valid tool to study the origin of the subsiding shell.

\section{Investigation of the vertical momentum budget terms}

In Fig. 1 the shell of descending air was clearly visible in both the observational data and in the LES results. To investigate the cause of this shell, we can benefit from the additional information gained from the simulations, such as the individual terms of the vertical momentum equation. Neglecting the Coriolis forcing, the vertical momentum equation used in LES can be split into the resolved advection terms (denoted as $A$ ), the buoyancy force $(B)$, the vertical pressure gradient $(P)$, and the parameterized unresolved subgrid diffusion $(S)$ :

$$
\begin{aligned}
\frac{\partial w}{\partial t}= & \overbrace{-u_{j} \frac{\partial w}{\partial x_{j}}+g \frac{\theta_{\boldsymbol{v}}-\overline{\theta_{v}}}{\Theta_{0}}}^{A}-\frac{1}{\rho_{0} \frac{\partial p^{\prime}}{\partial z}} \\
& \underbrace{P}_{S} \\
& \overbrace{\frac{\partial}{\partial x_{j}}\left[K_{m}\left(\frac{\partial u_{j}}{\partial z}+\frac{\partial w}{\partial x_{j}}\right)\right]}^{B} .
\end{aligned}
$$

Here $\theta_{v}$ denotes the virtual potential temperature, $\overline{\theta_{v}}$ the slab average virtual potential temperature, $\Theta_{0}$ a reference temperature, $\rho_{0}$ a reference density, $p^{\prime}$ the modified pressure, and $K_{m}(x, y, z)$ the subgrid-scale eddy viscosity.

One or more of these forcings should be responsible for the minimum in the $w$ profile around the cloud edge. If mechanical forcing is the main process behind the subsiding shell, the pressure gradient should be negative at the edge of the cloud. Evaporative cooling induced by horizontal mixing over the cloud edge, on the other hand, would result in a negative buoyancy force in the shell. The four terms are plotted in Fig. 2 as a function of the normalized distance to the center of the cloud $x / L_{c}$.

In these figures a strong minimum in buoyancy (upper-left graph) that appears just around the cloud is counteracted by the other terms. This means that buoyancy (and thus evaporative cooling) seems to drive the descending shell. This is in agreement with the findings of RDJ03, although they could not be conclusive with regard to the role of the other terms, in particular the pressure-gradient term. Indeed, looking at the upperright graph in Fig. 2, the simulations clearly indicate that the pressure gradient is not causing the shell, but, like the advection and the subgrid diffusion term, coun- teracts the downward acceleration. This shows unambiguously that the descending shell is driven by negative buoyancy, resulting from evaporative cooling following lateral mixing of environmental air with cloudy air.

It should be noted here that the terms $A, B, P$, and $S$, as shown in of Fig. 2, do not balance. This unbalance is caused by the conditional sampling over clouds with a fixed size. This follows from the fact that a growing cloud is associated with a positive acceleration. As the cloud keeps growing, however, it does not meet the size criterion of $L_{c}=400 \mathrm{~m}$ anymore and leaves the ensemble, thus causing an apparent unbalance in the vertical momentum budget.

\section{Analysis of BOMEX}

\section{a. Occurrence of the descending shell in BOMEX}

To investigate whether the descending shell is a specific feature of the SCMS case, or rather a more generic feature of shallow cumulus clouds, we analyze the BOMEX numerical case of marine shallow cumulus clouds in the same way as was done for SCMS. Since BOMEX is a steady-state case, a much larger time window for observations could be taken. Ten simulations of $12 \mathrm{~h}$ each have been done, of which each first $3 \mathrm{~h}$ were disregarded as spinup. Data from 1021 flights through clouds of $L_{c}=400 \mathrm{~m}$ at a measurement height of $1000 \mathrm{~m}$ have been collected; the results for $w, \theta_{l}, q_{t}$, and the vertical momentum terms are presented in Fig. 3. The shapes of the profiles in Fig. 3 resemble the SCMS results, including the existence of a descending shell in the $w$ profile. Also similar to the SCMS results, the shell is associated with an area of negative buoyancy (with a lateral size of 50-100 m), while the pressure gradient is again positive at cloud edge. Overall, this suggests that the descending shell due to evaporative cooling by lateral mixing is a generic feature of shallow cumulus clouds. It might be noted that the shell looks similar to artifacts due to the advection scheme of the LES. This is discussed in the appendix; the shell appears to be independent of the used advection scheme.

Looking at the location of the velocity minimum in both Fig. 1 and in Fig. 3, the subsiding shell seems to lie at the edge or just outside the cloud (signified by the vertical dashed line in the figures). Indeed, the buoyancy decreases within the cloud from cloud core value to its minimum virtually at the edge, which makes sense since the shell is associated with recently evaporated air.

The existence of these downdrafts and the associated shear also generate additional turbulence at the edge of 

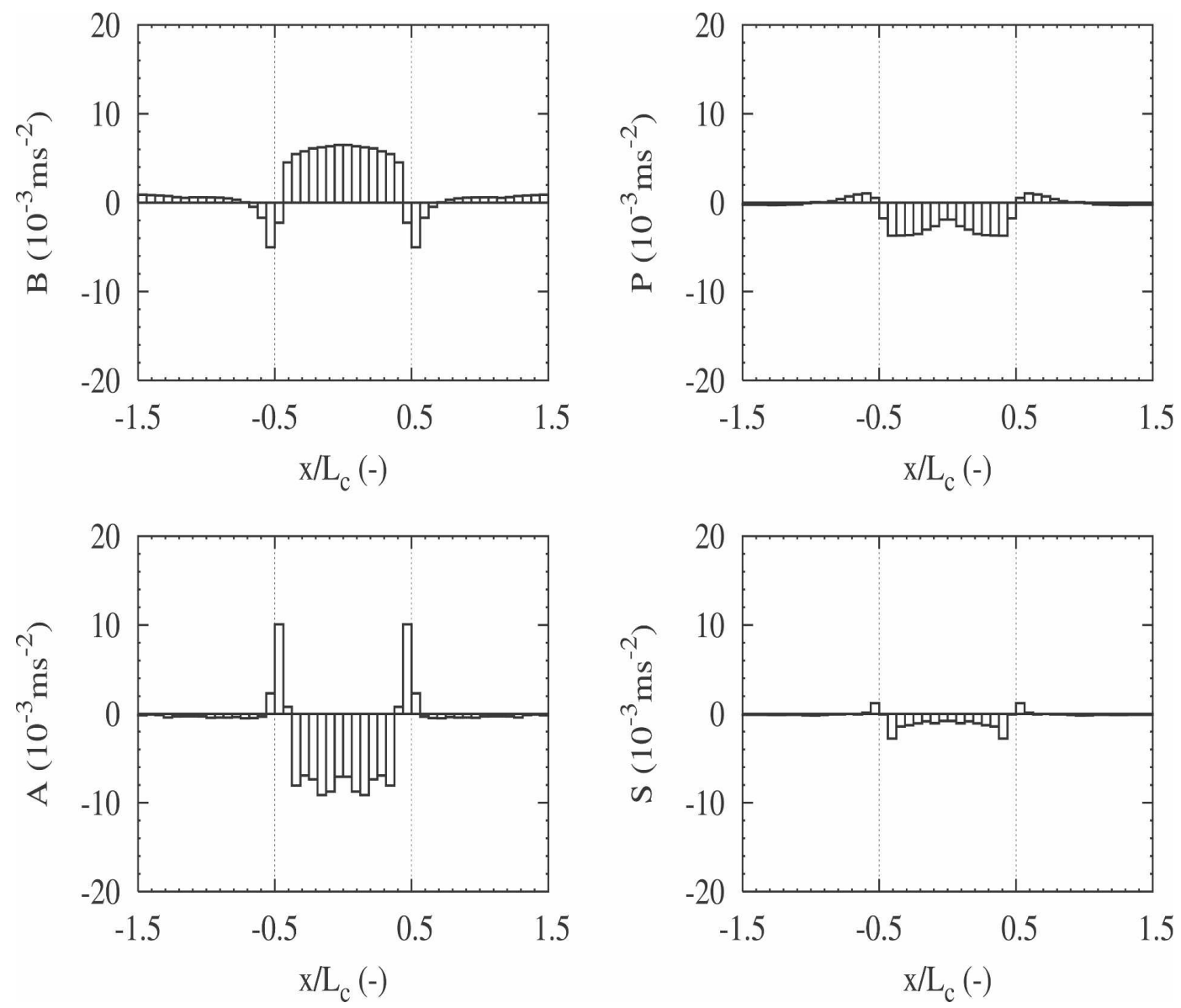

FIG. 2. The individual terms of the vertical momentum Eq. (1): (top left) buoyancy $B$, (top right) vertical pressure gradient $P$, (bottom left) advection $A$, and (bottom right) subgrid diffusion $S$, plotted against the distance to cloud center $x$ (normalized with cloud size $L_{c}$ ).

the cloud. For the BOMEX simulations, the turbulence dissipation rate $\varepsilon$ is plotted in Fig. 4. Similar to the observational results of Siebert et al. (2006), the shell is much more turbulent than the outer environment, and shows a maximum outside the cloud core. This can be expected, since this is the region of the largest gradients. The increased turbulence should result in a better (more homogeneous) mixing even before air is entrained into the cloud core.

\section{b. Mass flux through the shell}

Looking again at the relatively modest size of the subsiding shell in the $w$ profile in Fig. 3, we may wonder what the importance of the subsiding shell is on the interaction between the cloud and its environment. To illustrate the significance of the shell an instantaneous cross section of a cloud is shown in Fig. 5. Here, vectors denote the in-plane velocity, and the buoyancy excess is depicted in gray tones. Quite consistently, a minimum in buoyancy (the light area in Fig. 5) can be observed not only at cloud top but almost everywhere around cloud edge. Especially around the right side of the cloud the area with negative velocities is large. There, environmental air mixed into the cloud seems to be carried down before entering the cloud.

Moreover, it has to be kept in mind that these "flythrough" profiles are one-dimensional representations. The significance of that becomes clear when looking at the vertical mass flux $M$ for 400-m-sized clouds. On the assumption of a circular-shaped cloud, $M$ would be

$$
M(x) d x=\rho w(d x) 2 \pi x d x .
$$

The contribution to $M$ of the slowly moving air further away from cloud center cannot be neglected compared to the fast-moving cloud core, because of the significant area of the outer region. This is shown in Fig. 6: 10\% (the black area) of the air flowing upward through the cloud comes down directly through the region where 

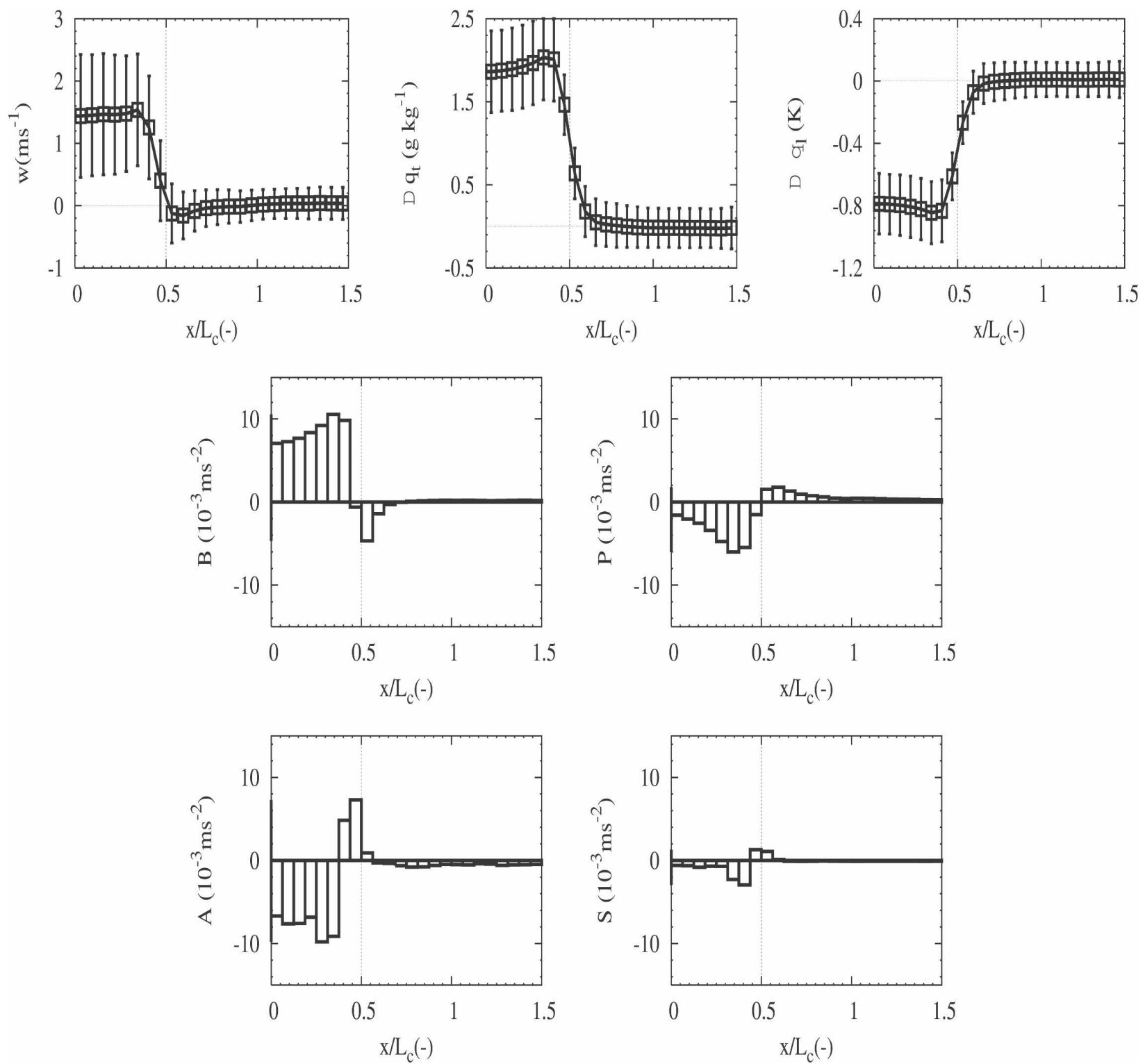

FIG. 3. BOMEX results: profiles of vertical velocity, total moisture, and liquid potential temperature and the vertical momentum budget terms (buoyancy, pressure gradient, advection, and subgrid diffusion) are plotted here for 1021 cloud transects of $400 \mathrm{~m}$ appearing in an ensemble average of 10 BOMEX runs of $12 \mathrm{~h}$ each. Fly-through level is $1000 \mathrm{~m}$.

$B<0$, while another $13 \%$ (the dark gray area) is dragged along downward with the shell, in total balancing almost a quarter of the in-cloud upflow. In Fig. 7 these effects are shown for various cloud sizes. In the left panel of Fig. 7 the total upward mass flux through the cloud and the total downward mass flux through the shell (defined here as the region of negative velocity directly adjacent to the cloud) is shown. For $L_{c}=400$ $\mathrm{m}$, the relative mass flux through the shell amounts to about a quarter, a value decreasing with cloud size. In Fig. 7 (right) the integrated amount of buoyancy in the cloud and in the shell is shown; here the negative inshell buoyancy is even dominating the positive in-cloud buoyancy for smaller clouds.

The picture that arises here is the following: since the environment has no direct interaction with the warm cloud core, but is fenced off by the shell, the environment actually "sees" the cloud as a negatively buoyant, often downward-moving entity. The significant amount of air dragged downward might also explain the results of J90. He observed significant amounts of air with properties equal to that of higher environmental levels. 


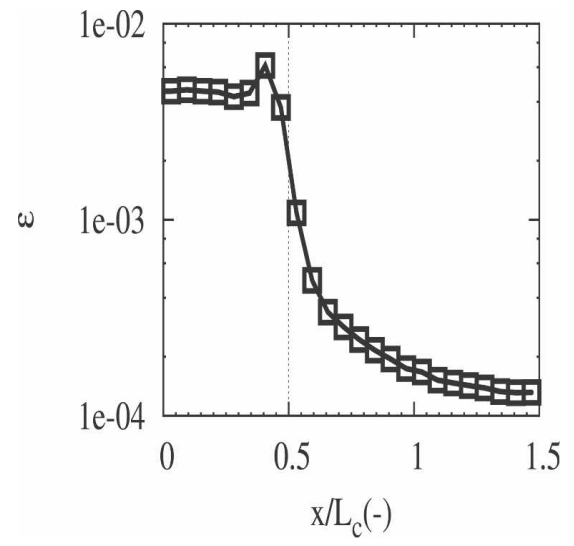

FIG. 4. The dissipation rate (on a log scale) profiles for BOMEX. For further explanation of the graph, see Fig. 3.

Since environmental air is first dragged downward by the subsiding shell before being mixed into the cloud, we can now see that this downward motion would cause a bias toward mixing at heights above actual mixing height.

The downward mass flux through the shell also has implications for the necessary compensating subsidence of the environment; these are addressed in section 6 .

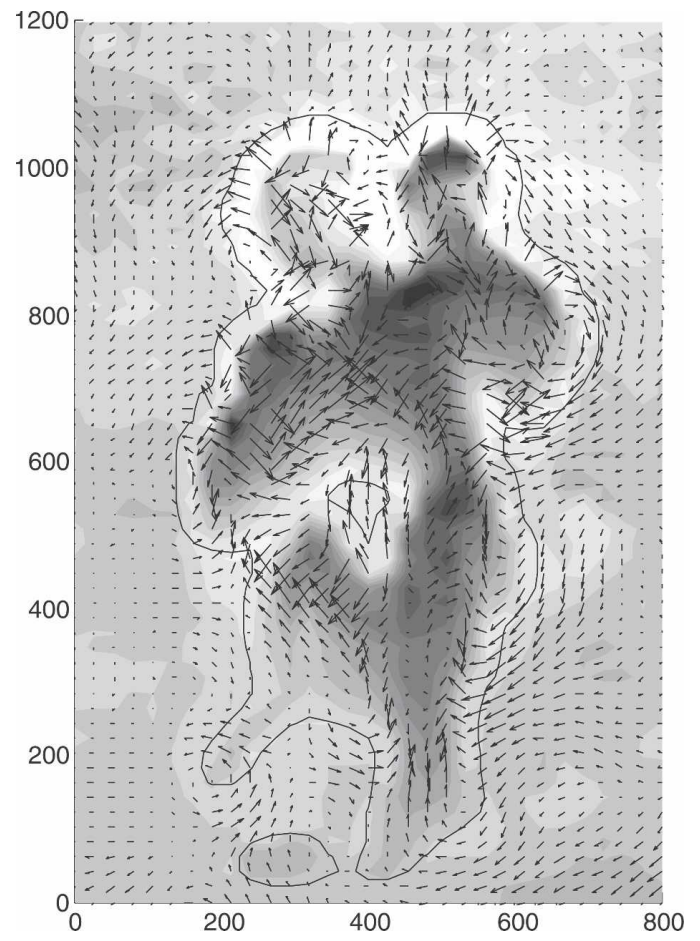

FIG. 5. A $y-z$ plane cross section (with distances in meters) through the center of mass of a cloud. Cloud edge is denoted by the black line, the vectors signify the in-plane velocity, and the virtual potential temperature excess is displayed in gray tones, with the lighter areas more negatively buoyant.

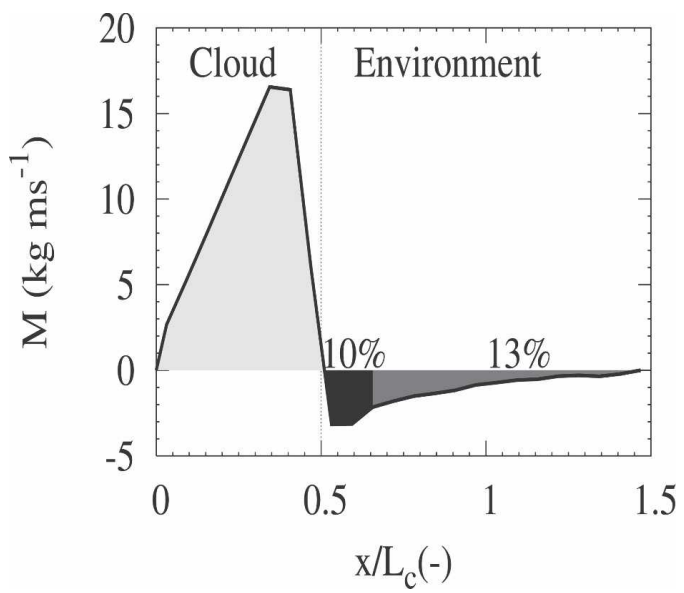

FIG. 6. The vertical mass flux $M$ through 400 -m-sized clouds as a function of the distance to cloud center $x$ for BOMEX at $1000-\mathrm{m}$ height. For small distances, $M$ goes to zero because of the small size of the area. The black colored area signifies the mass flux through the negatively buoyant shell (around $10 \%$ of the total cloud mass flux). The dark gray area is dragged downward induced by the shell, resulting in a total of $25 \%$ of the in-cloud upflow.

\section{c. Influence of mean wind and shear}

Exploration of the in-cloud values of the (thermo-) dynamical quantities as well as the vertical momentum budget terms should yield their maximum/minimum values in the cloud core. However, when looking in detail at Fig. 3, the profiles appear to be flat within the clouds, with even slightly decreasing values when approaching cloud center. In the SCMS results (Figs. 1 and 2) this effect appears to be much less pronounced. Indeed, if only transects through the BOMEX simulations are taken into account when taken from west to east (i.e., against the mean wind), the vertical velocity profile appears to be asymmetrical (see Fig. 8). Of course, adding all east-west transects to Fig. 8 would result in a symmetrical profile again, but for heavily skewed distributions the maximum would be out of cloud center. The other variables (not shown here) show a very similar skewness.

The only component in the system that breaks the lateral symmetry is the mean horizontal wind. For BOMEX this mean wind has a value of approximately $\mathbf{U} \approx[-10+(z / 555), 0] \mathrm{m} \mathrm{s}^{-1}$ in the cloud layer, which is much stronger than the mean horizontal wind in SCMS [where the geostrophic wind is equal to $(-4,4)$ $\mathrm{m} \mathrm{s}^{-1}$ ]. Since measurements are done in the reference frame of the cloud, which advects with the mean wind, this value should have no influence on the cloud vertical velocity profile. However, the vertical mean wind shear $\left[(\partial \mathbf{U} / \partial z) \approx(1.8,0) \mathrm{m} \mathrm{s}^{-1} \mathrm{~km}^{-1}\right.$ in BOMEX] might. In a strongly sheared environment the cloud 

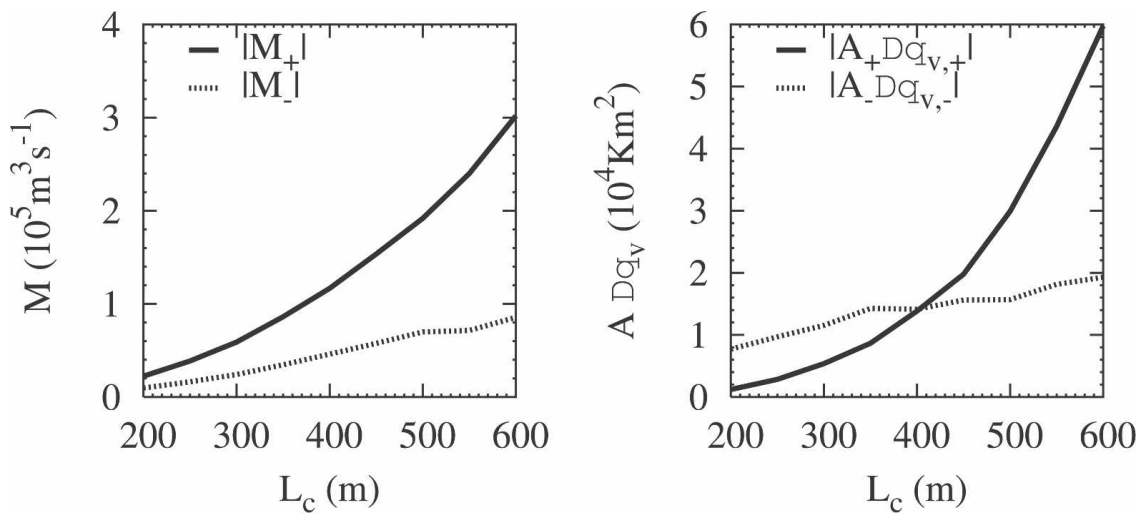

FIG. 7. The absolute values of the mass flux $M$ (left) and the integrated virtual potential temperature difference $A \Delta \theta_{v}$ through the cloud core and the shell. The + and - subscripts denote sampling over regions with positive and negative vertical velocity, respectively, and $A$ indicates the area of that region.

core is displaced from the center of the cloud (see Heymsfield et al. 1978). Later studies (e.g., Perry and Hobbs 1996) found that vertical shear is also often responsible for a halo of humid air up to several cloud radii away from the cloud edge on the downshear side (i.e., the right side of the cloud for positive values of the shear and vice versa). Laird (2005) found a similar increase in clear-air humidity, in a study on the SCMS cloud database, only in a smaller area around the cloud and without much preference for either the up- or downshear side of the cloud. This was blamed on the low amount of shear in the SCMS [being around $(\partial \mathbf{U} /$ $\left.\partial z) \approx(0,0.7) \mathrm{m} \mathrm{s}^{-1} \mathrm{~km}^{-1}\right]$.

Besides a physical explanation of the asymmetry, an argument of numerical nature should also be considered. In nature clouds would be advected by the mean wind with the same horizontal velocity as their environment. Since the statistics are taken in the reference frame of the cloud, mean wind advection in itself should not influence the results. In simulations, however, the discrete representation and all-or-nothing condensation scheme result in an environment moving with the mean wind while the cloud is effectively standing still, except for the instants when condensation level is reached adjacent to the cloud, moving the cloud into another grid box.

To investigate the effect of this artifact, it is useful to split the advection term $(A)$ of the vertical momentum Eq. (1) into a large-scale transport by mean wind $(L)$ and by fluctuations $(F)$ term:

$$
A=-u_{j} \frac{\partial w}{\partial x_{j}}=\underbrace{-\left(U_{j}-U_{J}^{\mathrm{T}}\right) \frac{\partial w}{\partial x_{j}}}_{L} \underbrace{-u_{j}^{\prime} \frac{\partial w}{\partial x_{j}}}_{F} .
$$

The influence of $L$ on the profiles-which should be zero-can be studied by variation of the Galilean trans-
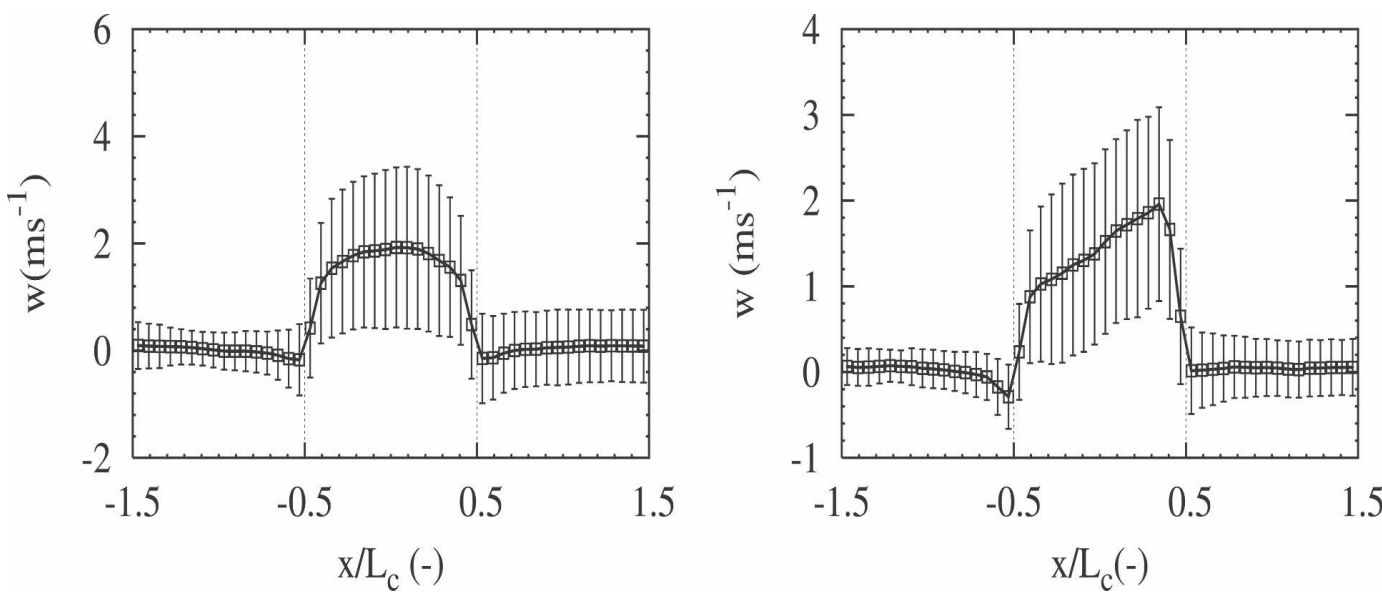

FIG. 8. The vertical velocity profile for (left) SCMS and (right) BOMEX, with all transects taken from west to east. 
TABLE 1. Simulations done to investigate the role of the mean horizontal wind. Based on BOMEX, Galilean transformation $U^{\mathrm{T}}$ and the mean velocity $U(z)$ were varied. Here $|(\partial u / \partial z)|$ was kept on the BOMEX value of $1 / 555 \mathrm{~s}^{-1}$, and a mean surface velocity was added to ensure $U(z)= \pm 1 \mathrm{~m} \mathrm{~s}^{-1}$ at the observational level of $1000 \mathrm{~m}$, yielding $U_{s}=(z / 555)-0.836$.

\begin{tabular}{ccc}
\hline \hline Name & $U^{\mathrm{T}}\left(\mathrm{m} \mathrm{s}^{-1}\right)$ & $U(z)\left(\mathrm{m} \mathrm{s}^{-1}\right)$ \\
\hline GA-2UM-1 & -2 & $-U_{s}$ \\
GA-1UM-1 & -1 & $-U_{s}$ \\
GA0UM-1 & 0 & $-U_{s}$ \\
GA-2UM0 & -2 & 0 \\
GA0-1M0 & -1 & 0 \\
GA0UM0 & 0 & 0 \\
GA1UM0 & 1 & 0 \\
GA2UM0 & 2 & 0 \\
GA0UM1 & 0 & $U_{s}$ \\
GA1UM1 & 1 & $U_{s}$ \\
GA2UM1 & 2 & $U_{s}$ \\
\hline
\end{tabular}

formation velocity $U_{j}^{\mathrm{T}}$, thus affecting the effective mean wind $U_{j}-U_{j}^{\mathrm{T}}$. To investigate the combined effects of these two (both shear and mean wind advection) mechanisms, a set of cases is designed and described in Table 1 . Based on the strongly sheared BOMEX case, the Coriolis force is ignored in these simulations to eliminate the influence of the Ekman spiral. The mean wind is varied in such a way that at the level of measurement $(1 \mathrm{~km}) U_{j}-U_{j}^{\mathrm{T}}$ is either $-1,0$, or $+1 \mathrm{~m} \mathrm{~s}^{-1}$, and the amount of shear is equal to the BOMEX value of $1.8 \mathrm{~m} \mathrm{~s}^{-1} \mathrm{~km}^{-1}$. To study the numerical effects, $U^{\mathrm{T}}$ is varied around $U(z=1000 \mathrm{~m})$ to see the effect of the mean wind advection.

The results of these simulations are plotted in Fig. 9. First of all, looking at the solid lines in the center column (i.e., neither mean wind nor shear), it can be seen that all profiles are symmetrical, indicating that the mean horizontal wind is indeed the cause of the asymmetry seen in Fig. 8. Focusing then on the variation in $U^{\mathrm{T}}$ depicted in the center column of this figure, the effect of the mean wind advection is clearly visible. Here $L$ peaks sharply at cloud edge, and this is where the buoyancy and velocity profiles are mainly affected. In extreme cases, the subsiding shell is completely filled in on the downwind side of the cloud, and the location of the in-cloud velocity maximum is also shifted to the downwind side. The total water content appears less skewed, since the scalar profiles are only indirectly affected by the vertical advection term $w(\partial \phi / \partial z)$.

By comparing the different columns in Fig. 9 the effect of vertical shear can be seen. On the downshear side (the left-hand side in the left column), there is a clear increase in humidity up to a cloud radius away from the edge, while steep gradients are observed at the upshear side of the cloud. Aside from yielding skewed profiles, this also greatly affects the existence of the shell, as is schematically shown in Fig. 10. On the downshear side the region with just evaporated air is increased, causing a wider shell; the lack of mixing and increased upward drag on the upshear side prevents the occurrence of a shell there. The cloud core (signified by maximum velocity, buoyancy, and humidity) remains located above cloud base, and its position is thus skewed toward the upshear side.

Looking at the combined effect of both the vertical shear and the mean wind advection, the shear is clearly dominant, since the sheared profiles do not change much when varying $U^{\mathrm{T}}$. One notable exception should however be made: the mean wind advection is capable of creating a small artificial shell of its own at the upwind, upshear side of the $w$ profile, helped by the sharp gradient of this profile, which originally was created by the shear.

\section{Three-layer model}

To get a better understanding on the role and behavior of the shell, below we develop a simple analytical model within the framework of Asai and Kasahara (1967, hereafter AK67). The AK67 model uses a cylindrical geometry and employs a "top hat" approach, dividing the cloud layer into two regions: a cloudy region with positive mass flux and around it a much larger environmental region with a small downward velocity, in total exactly compensating the cloud mass flux. We extend this model by adding a third region between cloud and environment, that is, a region for the shell with its own velocity (see Fig. 11). The present model is also a significant simplification of the model by AK67, since we discard the vertical dimension, and assume a steady state. As such the three-layer model is most representative of the middle of the cloud layer. In any case, it is not to be expected that this approach yields a solid quantitative model for the entire cloud layer, yet it may yield a qualitative description of the shell, giving understanding of the consequences of a system driven by buoyancy that experiences significant shear at the edges and therefore lateral mixing of mass and momentum. Specifically, it is of interest to see whether a cloud exists in a realistic way if the cloud dynamics are completely dominated by lateral mixing through the shell.

Assuming rotational symmetry, Eq. (1) can be rewritten into

$$
\frac{\partial w}{\partial t}=-\frac{1}{r} \frac{\partial}{\partial r}(r u w)-\frac{\partial}{\partial z}(w w)+\frac{g}{\Theta_{0}}\left(\theta_{v}-\bar{\theta}_{v}\right)=0,
$$



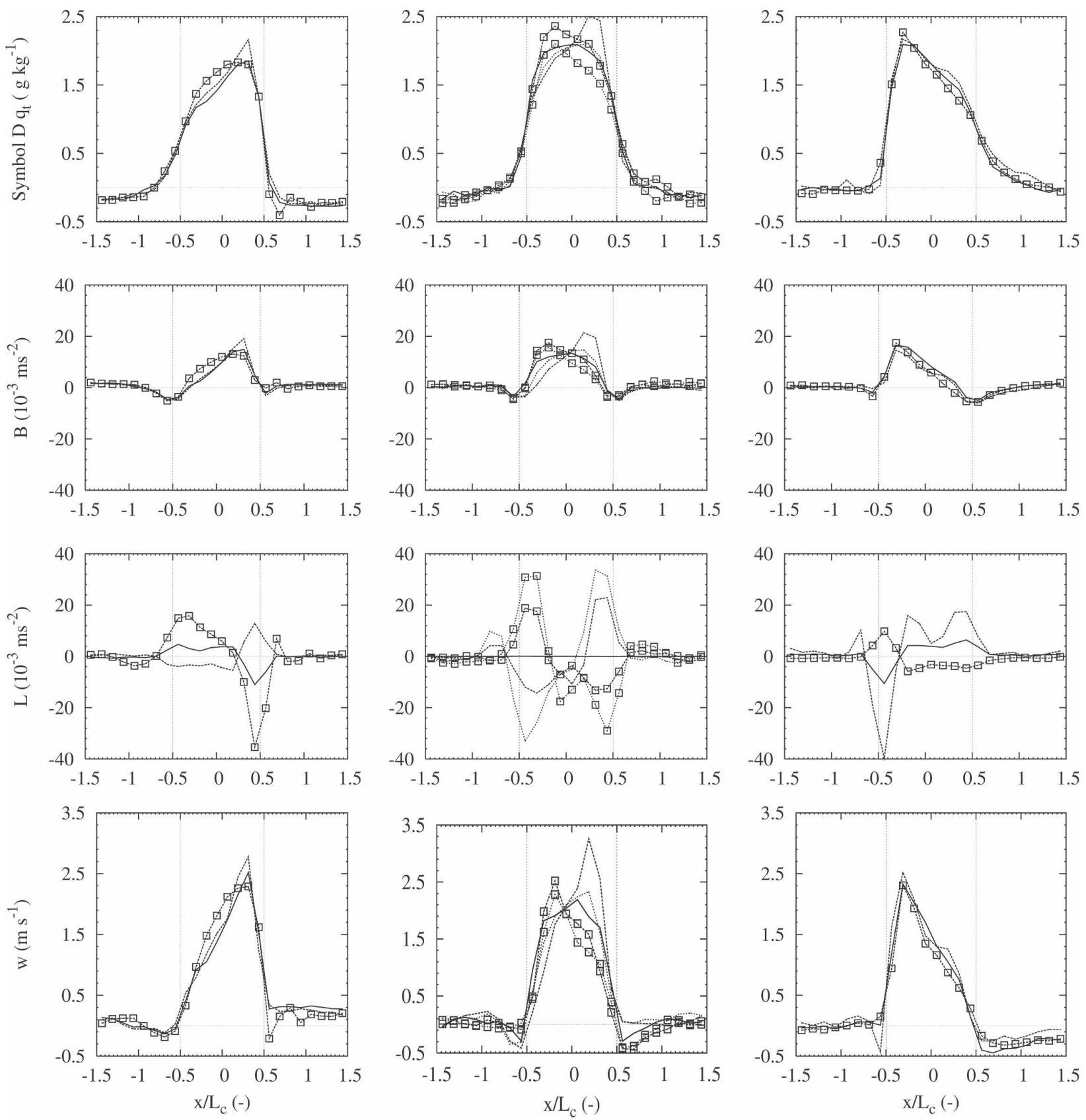

$$
\begin{aligned}
& \mathrm{u}_{\mathrm{T}}=-2 \mathrm{~ms}^{-1}, \mathrm{u}=-\mathrm{u}_{\mathrm{s}} \\
& -\mathrm{u}_{\mathrm{T}}=-1 \mathrm{~ms}^{-1}, \mathrm{u}=-\mathrm{u}_{\mathrm{s}} \\
& -\mathrm{u}_{\mathrm{T}}=0 \mathrm{~ms}^{-1}, \mathrm{u}=-\mathrm{u}_{\mathrm{s}}
\end{aligned}
$$$$
\begin{aligned}
& \cdots \cdots \mathrm{u}_{\mathrm{T}}=-2 \mathrm{~ms}^{-1}, \mathrm{u}=0 \mathrm{~ms}^{-1} \\
& \cdots \mathrm{u}_{\mathrm{T}}=-1 \mathrm{~ms}^{-1}, \mathrm{u}=0 \mathrm{~ms}^{-1} \\
&-\mathrm{u}_{\mathrm{T}}=0 \mathrm{~ms}^{-1}, \mathrm{u}=0 \mathrm{~ms}^{-1} \\
& \because \cdot \mathrm{u}_{\mathrm{T}}=1 \mathrm{~ms}^{-1}, \mathrm{u}=0 \mathrm{~ms}^{-1} \\
& \cdots \mathrm{u}_{\mathrm{T}}=2 \mathrm{~ms}^{-1}, \mathrm{u}=0 \mathrm{~ms}^{-1}
\end{aligned}
$$$$
\begin{aligned}
\mathrm{u}_{\mathrm{T}}=0 \mathrm{~ms}^{-1}, \mathrm{u}=\mathrm{u}_{\mathrm{s}} \\
-\mathrm{u}_{\mathrm{T}}=1 \mathrm{~ms}^{-1}, \mathrm{u}=\mathrm{u}_{\mathrm{S}} \\
\because \quad \mathrm{u}_{\mathrm{T}}=2 \mathrm{~ms}^{-1}, \mathrm{u}=\mathrm{u}_{\mathrm{s}}
\end{aligned}
$$

FIG. 9. The influence of shear (left column) $U(z)=-U_{s}(z)$, (center) $U_{z}=0$, (right column) $U(z)=-U_{s}(z)$; and the Galilean transform varied within the graphs on (from top to bottom) the total water content $q_{t}$, the buoyancy $B$, the large-scale horizontal advection $L$, and the vertical velocity $w$. 


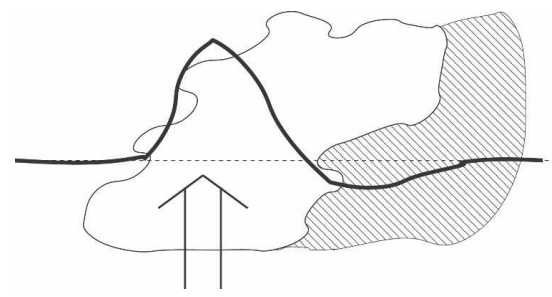

FIG. 10. The conceptual picture of a cloud tilted because of vertical shear, including its humidity halo (shaded area). The vertical velocity profile is sketched on top of the cloud. Since the cloud core is less skewed than the rest of the cloud, this results in an upshear location of the velocity maximum. The humidity halo ensures a wide and deep subsiding shell on the downshear side of the cloud.

and the continuity equation into

$$
\frac{1}{r} \frac{\partial}{\partial r}(r u)+\frac{\partial}{\partial z}(w)=0 .
$$

Integrating Eq. (5) over the region $n \in\{1,2,3\}$ with area $A_{n}=\pi\left(r_{n}^{2}-r_{n-1}^{2}\right)$, and disregarding vertical gradients as mentioned, after dividing by $2 \pi$ we get

$$
\int_{r_{n-1}}^{r_{n}}\left[\frac{1}{r} \frac{\partial}{\partial r}\left(r u_{n}\right)\right] r d r=r_{n} \tilde{u}^{n}-r_{n-1} \tilde{u}^{n-1}=0,
$$

where $\tilde{u}^{n}$ denotes the average of $u$ over the circle with radius $r_{n}$, that is, the boundary between region $n$ and $n+1$. With $r_{0}=0$ this gives $\tilde{u}^{n}=0$ for all $n>0$; a direct consequence of the absence of vertical gradients in the flow. Integration of Eq. (4) over area $A_{n}$ yields

$$
2 \pi r_{n} \widetilde{u w}^{n}-2 \pi r_{n-1} \widetilde{u w}^{n-1}=A_{n} \frac{g}{\Theta_{0}}\left(\Delta \bar{\theta}_{v}^{n}\right),
$$

with $\Delta{\overline{\theta_{v}}}^{n}={\overline{\theta_{v}}}^{n}-\bar{\theta}_{v}$, where ${\overline{\theta_{v}}}^{n}$ denotes the average $\theta_{v}$ over the area $A_{n}$. Decomposing the boundary covariance term into $\widetilde{u w}^{n}=\tilde{u}^{n} \tilde{w}^{n}+\widetilde{u^{\prime} w^{\prime n}}$, and using $\tilde{u}^{n}=$ 0 , we only need to account for the covariance of fluctuations at the boundary, that is, the effect of turbulent mixing at the boundary. Rather than working with the areas $A_{n}$, we will use the relative area coverage $\sigma_{n}=$ $A_{n} /\left(A_{1}+A_{2}+A_{3}\right)$, and use the cloud area as the reference $A_{1}=\pi r_{1}^{2}$. Substituting $A_{n}=\pi r_{1}^{2} \sigma_{n} / \sigma_{1}$, we obtain

$$
\begin{gathered}
\frac{r_{1}^{2}}{2} \frac{g \Delta{\overline{\theta_{v}}}^{1}}{\Theta_{0}}=r_{1} \widetilde{u^{\prime} w^{\prime \prime}}, \\
\frac{\sigma_{2}}{\sigma_{1}} \frac{r_{1}^{2}}{2} \frac{g \Delta \bar{\theta}_{v}^{2}}{\Theta_{0}}=-r_{1} \widetilde{u^{\prime} w^{\prime 1}}+r_{2} \widetilde{u^{\prime} w^{\prime 2}}, \\
\frac{\sigma_{3}}{\sigma_{1}} \frac{r_{1}^{2}}{2} \frac{g \Delta \bar{\theta}_{v}^{3}}{\Theta_{0}}=-r_{2} \widetilde{u^{\prime} w^{\prime 2}},
\end{gathered}
$$

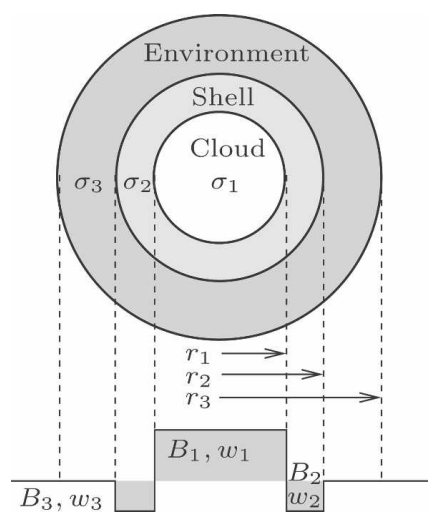

FIG. 11. The proposed model divides the cloud layer in three shells: in the center the cloud core, with positive vertical velocity and buoyancy; wrapped around the core lies the subsiding shell with negative vertical velocity and buoyancy, and finally an environmental region balancing the other two.

while conservation laws dictate

$$
\begin{aligned}
\sigma_{1}+\sigma_{2}+\sigma_{3} & =1, \\
\sigma_{1} \bar{w}^{1}+\sigma_{2} \bar{w}^{2}+\sigma_{3} \bar{w}^{3} & =0, \\
\sigma_{1} \Delta{\overline{\theta_{v}}}^{1}+\sigma_{2} \Delta{\overline{\theta_{v}}}^{2}+\sigma_{3} \Delta{\overline{\theta_{v}}}^{3} & =0,
\end{aligned}
$$

where $\bar{w}^{n}$ represents the area-averaged velocity of region $n$. Physically, Eqs. (8)-(10) express that the buoyancy force is counteracted by the turbulent mixing of momentum over the boundaries. By this mechanism a balance is reached, allowing the velocities to reach an equilibrium state. The shell region experiences mixing at both boundaries. Following AK67, we apply Prandtl mixing length theory to the turbulent mixing terms

$$
\widetilde{u^{\prime} w^{\prime n}}=-\left.K_{n} \frac{d w}{d r}\right|_{r=r_{n}} \rightarrow-K_{n} \frac{\bar{w}^{n+1}-\bar{w}^{n}}{\frac{1}{2}\left(r_{n+1}-r_{n-1}\right)},
$$

with, for $K_{n}$,

$$
K_{n}=\ell^{2}\left|\frac{\bar{w}^{n+1}-\bar{w}^{n}}{\frac{1}{2}\left(r_{n+1}-r_{n-1}\right)}\right| .
$$

For the mixing length $\ell$ it seems reasonable to assume that the width of the shell is the relevant length scale,

$$
\ell=\kappa\left(r_{2}-r_{1}\right)
$$

with von Kármán constant $\kappa=0.4$. We introduce the relative shell size $\zeta=\left(r_{2}-r_{1}\right) / r_{1}$ as a model parameter, and, with $f(w)=w|w|$, rewrite Eqs. (8) and (9) into 


$$
\begin{aligned}
& \sigma_{1} \frac{g \Delta{\overline{\theta_{v}}}^{1}}{\Theta_{0}}=\frac{8 \kappa^{2}}{r_{1}} \frac{\sigma_{1} \zeta^{2}}{(1+\zeta)^{2}} f\left(\bar{w}^{1}-\bar{w}^{2}\right), \\
& \sigma_{2} \frac{g \Delta \bar{\theta}_{v}^{2}}{\Theta_{0}}=-\sigma_{1} \Delta{\overline{\theta_{v}}}^{1}+\frac{8 \kappa^{2}}{r_{1}} \frac{\sigma_{1}^{2} \zeta^{2}(1+\zeta)}{\left(1-\sqrt{\sigma_{1}}\right)^{2}} f\left(\bar{w}^{2}-\bar{w}^{3}\right),
\end{aligned}
$$

which need be solved together with Eqs. (11)-(13). Note that $\sigma_{2}$ can be expressed in terms of $\sigma_{1}$ and $\zeta$ : $\sigma_{2}=\sigma_{1}\left(\zeta^{2}+2 \zeta\right)$. The environmental velocity $\bar{w}^{3}$ follows from Eq. (12). A further simplification can be obtained by studying the equations in dimensionless form, where we rescale the velocities by the buoyancy velocity scale $W=\sqrt{g \Delta \bar{\theta}_{v}^{1}\left(2 r_{1}\right) / \Theta_{0}}$, commonly used in Rayleigh-Benard convection. From the LES data we know that $W$ is of the order unity; for example, for clouds with size $L_{c}=2 r_{1}=400 \mathrm{~m}$ we find $W \approx 2 \mathrm{~m} \mathrm{~s}^{-1}$. Using $w_{n}=\bar{w}^{n} / W$ and introducing the parameter $b$, which represents the average buoyancy in the shell relative to the average buoyancy in the cloud; that is, $b={\overline{\theta_{v}}}^{2} \bar{\theta}_{v}{ }^{1}$, we get

$$
\begin{gathered}
1=\frac{(4 \kappa \zeta)^{2}}{(1+\zeta)^{2}} f\left(w_{1}-w_{2}\right), \\
1+b\left(\zeta^{2}+2 \zeta\right)=\frac{(4 \kappa \zeta)^{2}(1+\zeta) \sigma_{1}}{\left(1-\sqrt{\sigma_{1}}\right)^{2}} f\left(w_{2}-w_{3}\right) .
\end{gathered}
$$

We can now solve the equations and derive expressions for $w_{1}$ and $w_{2}$ in terms of the parameters $b$ and $\zeta$, the relative buoyancy, and the relative size of the shell, respectively. Using Eq. (19) we immediately arrive at

$$
w_{1}=w_{2}+\frac{1+\zeta}{4 \kappa \zeta} \text {. }
$$
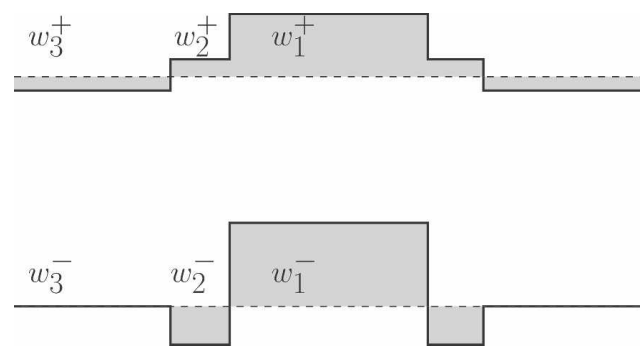

FIG. 12. The two distinct types of solutions resulting from the conceptual model: (top) solution $w_{2}^{+}$, the velocity in the shell lies between the cloud velocity and the environmental velocity; (bottom) solution $w_{2}^{-}$, the shell velocity is significantly lower than the environmental velocity.

Substituting this expression in Eq. (20), an expression for $w_{2}$ can be found analytically, which gives rise to two distinct types of solutions. These are depicted schematically in Fig. 12: the first solution (top panel) is the one that would be expected to arise when disregarding evaporative cooling, or when the upward force due to shear with the in-cloud updraft would be dominant; then the shell velocity would be somewhere between the mean cloud velocity and the velocity of the environment: $w_{1}>w_{2}>w_{3}$. The solution depicted in the bottom panel is the one that actually appears to arise in observations and in LES, with a distinct negative velocity in the shell; that is, $w_{2}<w_{3}$. The exact expressions are still rather involved, but since the cloud cover $\sigma_{1}$ is small (we take $\sigma_{1}=0.05$ ), approximate but accurate and more transparent expressions can be achieved by assuming $w_{3}=0$. This is justified since $w_{1}=O(1)$, $w_{2}=O(1)$, whereas $w_{3}=O\left(\sigma_{1}\right)$. Note that this does suggest an independence of the solution from the exact value of $w_{3}$. In this way we arrive at

$$
w_{2}=\left\{\begin{array}{llc}
w_{2}^{+}=+\frac{1}{4 \kappa \zeta} \sqrt{\frac{1+b\left(\zeta^{2}+2 \zeta\right)}{(1+\zeta) \sigma_{1}}} & \text { if } & b \geq b^{+}(\zeta), \\
w_{2}^{-}=-\frac{1}{4 \kappa \zeta} \sqrt{-\frac{1+b\left(\zeta^{2}+2 \zeta\right)}{(1+\zeta) \sigma_{1}}} & \text { if } & b^{-}(\zeta)<b \leq b^{+}(\zeta),
\end{array}\right.
$$

with

$$
\begin{aligned}
& b^{+}(\zeta)=-\frac{1}{\zeta^{2}+2 \zeta}, \\
& b^{-}(\zeta)=-\frac{1+(1+\zeta)^{3} \sigma_{1}}{\zeta^{2}+2 \zeta} .
\end{aligned}
$$

The corresponding cloud velocity solutions $w_{1}^{ \pm}$follow from Eq. (21). In Fig. 13 we have plotted the solutions for $w_{1}$ and $w_{2}$ as a function of the shell buoyancy $b$ for a fixed relative shell size of $\zeta=0.5$. The interesting point about the graphs and the structure of the solutions is that the descending shell solution $w_{2}^{-}$can only occur if the relative buoyancy and shell size are just right; that is, $b$ must lie between $b^{-}(\zeta)$ and $b^{+}(\zeta)$. For $b>b^{+}(\zeta)$, the shell (negative) buoyancy is not sufficient for the air to descend and the system settles into the solution $w_{2}^{+}$; on the other hand, if $b<b^{-}(\zeta)$, the shell buoyancy is so negative that the system enters into the unphysical state where $w_{1}<w_{3}$; that is, the shell 


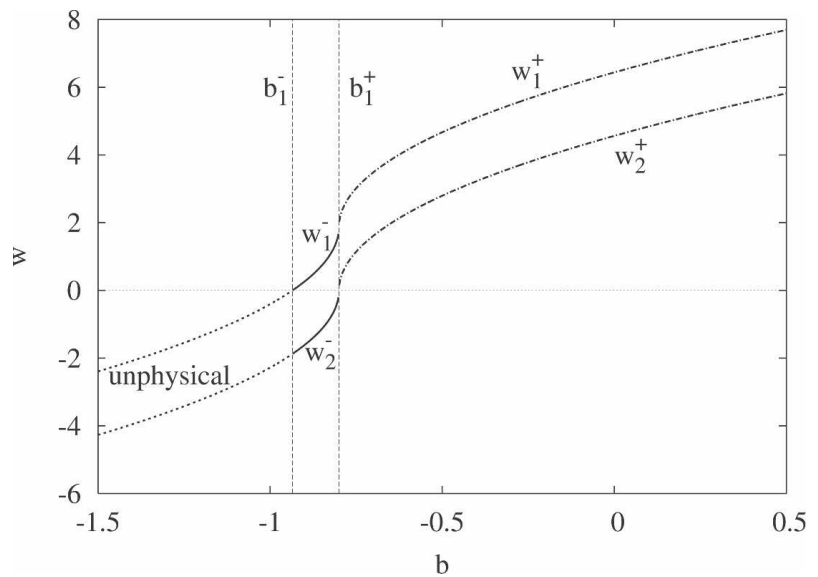

FIG. 13. Dimensionless cloud velocity $w_{1}$ and shell velocity $w_{2}$ as a function of the relative buoyancy $b$ in the shell, for a fixed relative shell size of $\zeta=0.5$. Depending on the value of $b$ the system settles either into solution $w^{+}$(dash-dotted lines: shell velocity between that of the cloud and the environment), or the solution $w^{-}$(solid lines: shell velocity smaller than the environmental velocity).

drags the entire cloud down. Hence the value of the shell buoyancy is rather a subtle parameter. This observation also holds for other values of the relative shell size, as can be seen in the phase diagram (Fig. 14), where we have indicated the occurrence of the solutions in the parameter space, that is, the $(\zeta, b)$ plane. Clearly the region pertaining to the descending shell solution is rather small.

Of course we would wish to elaborate the model such that it is able to actually predict the buoyancy in the shell; to this end the transport of moisture $q_{t}$ and temperature $\theta_{l}$ ought to be incorporated in the model, after which the buoyancy in the shell could be calculated by mixing cloudy properties (region 1) with environmental properties (region 3 ) into region 2. While such an approach is certainly feasible, we feel that at this stage such an extension would complicate the present model too much. The general conclusion from the present simple model is that the descending shell solution is an admissible mode within the system, but also a rather unlikely mode when compared to the rising shell mode. Apparently it can only occur as a result of a delicate balance between the shell size, the (negative) shell buoyancy, and the cloud fraction. This creates a new puzzle as to why the descending shell is nevertheless the preferred mode in observations and LES.

\section{Conclusions}

In this study, conditionally averaged results of largeeddy simulations of shallow cumulus clouds were com-

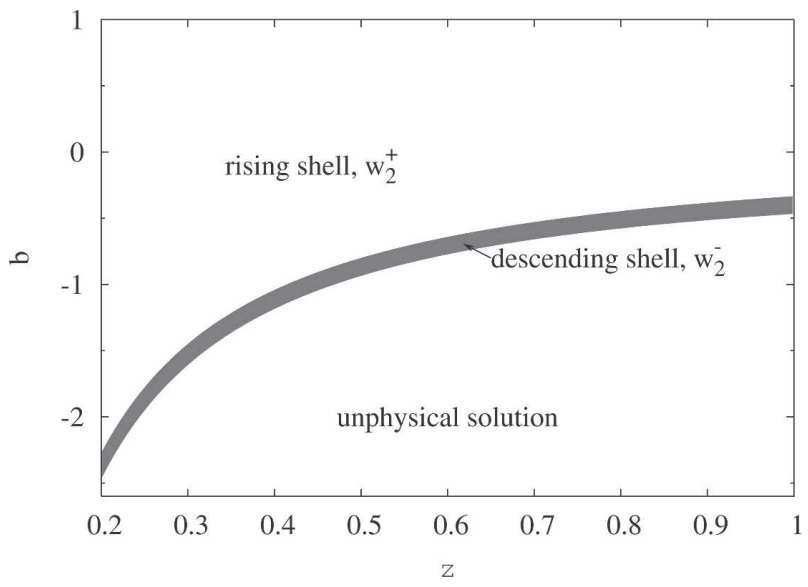

FIG. 14. Phase diagram: the regions of existence of the solutions are indicated in the parameter space $(\zeta, b)$. The region pertaining to the descending shell solution is remarkably small.

pared with observational results of Rodts et al. (2003) by focusing on the dynamical properties of the cloud. It was found that the simulations generate clouds with velocity, temperature, and moisture profiles very similar to the observations. Several important features of the clouds in observations were present in the simulations as well, in particular the descending air mass surrounding the cloud; they were investigated in detail in the LES.

Investigation of the individual terms of the vertical momentum equation showed that buoyancy is the dominant force in the shell, suggesting that evaporative cooling through lateral mixing over the cloud edge is the mechanism responsible for the shell. No evidence could be found for a role throughout the entire cloud layer for mechanical forcing; this should have resulted in a significant negative pressure-gradient force, which instead was found to be positive and thus even opposing the downdraft.

While around cloud top downdrafts can also be caused by overshoots in the form of a crownlike shape around the cloud edge, evaporative cooling ensures a consistent existence of the shell at all but the lowest levels of the cloud. Interestingly, this implies that, from the point of view of the environment, the cloud is perceived as a negatively buoyant, downward-moving entity. Moreover, the vertical mass flux through the shell appears to be significant compared with upward mass flux in the cloud core; this is especially true for smaller clouds, which are the most numerous.

Since the shell drags along a significant amount of environmental air downward and entrains it into the cloud, this might reconcile the concept of lateral mixing with observations where the in-cloud air appears to originate from higher environmental levels. Since the 
shell constantly refreshes the air at cloud edge, this could enhance evaporative cooling, ultimately creating a stronger shell.

In the simulations we observed that the lateral cloud profiles are skewed by the mean horizontal wind. Partly, this can be attributed to the discrete grid of the numerical simulations, but this is mostly due to the vertical shear of the horizontal mean wind. This vertical shear creates not only a displacement of the cloud core away from the cloud center, but is also responsible for a humid shadow or halo on the downshear side of the cloud, as seen before in observations. Since this shear results in reduced mixing at the upshear side of the cloud while enhancing mixing at the downshear side, this yields an asymmetry in the appearance and size of the shell.

The behavior of the shell was illustrated by a simple three-layer model analogous to the model by Asai and Kasahara (1967) where buoyancy is balanced by lateral mixing. The model showed that the descending shell is indeed one of the possible realizations in the system, but that it can only occur when the shell buoyancy and the shell size are well tuned to each other. The other model realization, which is more in line with the concept of lateral mixing of scalar quantities and of momentum, displays air around the cloud being dragged along and rises with a velocity between that of the cloud and environment. This solution of the model appeared to be much less critical. This makes the occurrence of the descending shells in observations and LES even more surprising.

Acknowledgments. The authors thank Maarten van Reeuwijk, Stephan de Roode, Evgeni Fedorovich, and Bjorn Stevens for the fruitful discussions and illuminating views. The investigations were supported by the Netherlands Organization for Scientific Research (NWO). This work was sponsored by the National Computing Facilities Foundation (NCF) for the use of supercomputer facilities.

\section{APPENDIX}

\section{Advection Scheme}

Besides the physical explanations for the existence of the shell given above, it could be argued that the occurrence in LES could also be due to numerical artifacts. For instance, the nonmonotonous central differencing advection scheme could cause numerical overshoots (wiggles) at the cloud edge. Such wiggles would look very similar to a subsiding shell-especially since the extent of the shell is only a few grid points. To rule
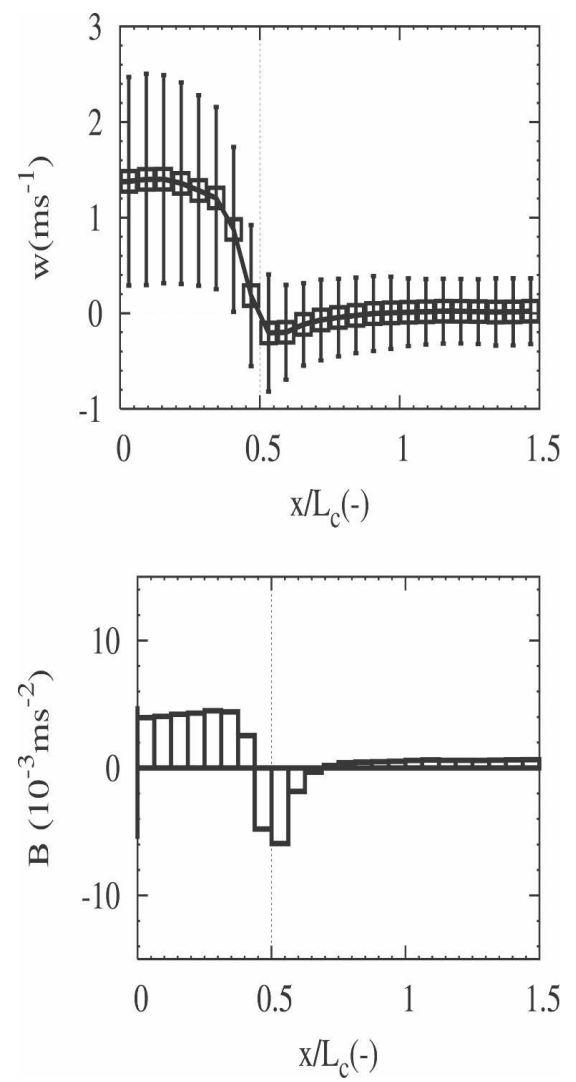

FIG. A1. Vertical velocity and buoyancy profile obtained from simulations identical with those displayed in Fig. 3, save for a monotonous instead of a nonmonotonous advection scheme.

out this possible cause another 11-h BOMEX run is done with the monotonous third order upwind kappa advection scheme (see Hundsdorfer et al. 1995). From the $L_{c}=400-\mathrm{m}$-sized clouds present in the final $8 \mathrm{~h}$ of this simulation, fly-through profiles were again collected at a height of $1000 \mathrm{~m}$. This yields for the vertical velocity and the buoyancy the results displayed in Fig. A1. Comparing Fig. A1 with Fig. 3, a similar shell can be observed for both schemes, meaning that the shell is not a result of overshoots depending on the used advection scheme.

\section{REFERENCES}

Asai, T., and A. Kasahara, 1967: A theoretical study of the compensating downward motions associated with cumulus clouds. J. Atmos. Sci., 24, 487-496.

Austin, P. H., M. B. Baker, A. M. Blyth, and J. B. Jensen, 1985: Small-scale variability in warm continental cumulus clouds. $J$. Atmos. Sci., 42, 1123-1138.

Betts, A. K., 1982: Saturation point analysis of moist convective overturning. J. Atmos. Sci., 39, 1484-1505.

Blyth, A. M., 1993: Entrainment in cumulus clouds. J. Appl. Meteor., 32, 626-641. 
_ W. A. Cooper, and J. B. Jensen, 1988: A study of the source of entrained air in Montana cumuli. J. Atmos. Sci., 45, 39443964.

Chlond, A., and A. Wolkau, 2000: Large-eddy simulation of a nocturnal stratocumulus-topped marine atmospheric boundary layer: An uncertainty analysis. Bound.-Layer Meteor., 95, $31-55$.

Cuijpers, J. W. M., and P. Duynkerke, 1993: Impact of skewness and nonlocal effects on scalar and buoyancy fluxes in convective boundary layers. J. Atmos. Sci., 55, 151-162.

French, J. R., G. Vali, and R. D. Kelly, 1999: Evolution of small cumulus clouds in Florida: Observations of pulsating growth. Atmos. Res., 52, 143-165.

Heymsfield, A. J., P. N. Johnson, and J. E. Dye, 1978: Observations of moist adiabatic ascent in northeast Colorado cumulus congestus clouds. J. Atmos. Sci., 35, 1689-1703.

Holland, J. Z., and E. M. Rasmusson, 1973: Measurements of the atmospheric mass, energy, and momentum budgets over a 500-kilometer square of tropical ocean. Mon. Wea. Rev., 101, 44-57.

Hundsdorfer, W., B. Koren, M. van Loon, and J. G. Verwer, 1995: A positive finite-difference advection scheme. J. Comput. Phys., 117, 35-46.

Jensen, J. B., P. H. Austin, M. B. Baker, and A. M. Blyth, 1985: Turbulent mixing, spectral evolution and dynamics in a warm cumulus cloud. J. Atmos. Sci., 42, 173-192.

Jonas, P., 1990: Observations of cumulus cloud entrainment. Atmos. Res., 25, 105-127.

Knight, C. A., and L. J. Miller, 1998: Early radar echoes from small, warm cumulus: Bragg and hydrometeor scattering. $J$. Atmos. Sci., 55, 2974-2992.

Kuang, Z., and C. S. Bretherton, 2006: A mass-flux scheme view of a high-resolution simulation of a transition from shallow to deep cumulus convection. J. Atmos. Sci., 63, 1895-1909.

Laird, N. F., 2005: Humidity halos surrounding small cumulus clouds in a tropical environment. J. Atmos. Sci., 62, 34203425.

Lu, M.-L., J. Wang, A. Freedman, H. H. Jonsson, R. C. Flagan, R. A. McClatchey, and J. H. Seinfeld, 2003: Analysis of humidity halos around trade wind cumulus clouds. J. Atmos. Sci., 60, 1041-1059.

Neggers, R., P. Duynkerke, and S. Rodts, 2003: Shallow cumulus convection: A validation of large-eddy simulation against air- craft and Landsat observations. Quart. J. Roy. Meteor. Soc., 129, 2671-2696.

Paluch, I. R., 1979: The entrainment mechanism in Colorado cumuli. J. Atmos. Sci., 36, 2467-2478.

Perry, K. D., and P. V. Hobbs, 1996: Influences of isolated cumulus clouds on the humidity of their surroundings. J. Atmos. Sci., 53, 159-174.

Reuter, G. W., and M. K. Yau, 1987a: Mixing mechanisms in cumulus congestus clouds. Part II: Numerical simulations. $J$. Atmos. Sci., 44, 798-827.

, and _- 1987b: Mixing mechanisms in cumulus congestus clouds. Part I: Observations. J. Atmos. Sci., 44, 781-797.

Rodts, S. M. A., P. G. Duynkerke, and H. J. J. Jonker, 2003: Size distributions and dynamical properties of shallow cumulus clouds from aircraft observations and satellite data. J. Atmos. Sci., 60, 1895-1912.

Siebert, H., K. Lehmann, M. Wendisch, and R. Shaw, 2006: Smallscale turbulence in clouds. Preprints, 12th Conf. on Cloud Physics, Madison, WI, Amer. Meteor. Soc., 12.1.

Siebesma, A. P., and J. W. M. Cuijpers, 1995: Evaluation of parametric assumptions for shallow cumulus convection. J. Atmos. Sci., 52, 650-666.

— cloud boundaries. Phys. Rev. Lett., 85, 214-217.

— son study of shallow cumulus convection. J. Atmos. Sci., 60, 1201-1219.

Squires, P., 1958: The microstructure and colloidal stability of warm clouds. Part 1: The relation between structure and stability. Tellus, 10, 256-261.

Stevens, B., and Coauthors, 2001: Simulations of trade wind cumuli under a strong inversion. J. Atmos. Sci., 58, 1870-1891.

Stommel, H., 1947: Entrainment of air into a cumulus cloud. $J$. Meteor., 4, 91-94.

Taylor, G. R., and M. B. Baker, 1991: Entrainment and detrainment in cumulus clouds. J. Atmos. Sci., 48, 112-121.

Telford, J. W., and P. B. Wagner, 1980: The dynamical and liquid water structure of the small cumulus as determined from its environment. Pure Appl. Geophys., 118, 935-952.

Zhao, M., and P. H. Austin, 2005: Life cycle of numerically simulated shallow cumulus clouds. Part I: Transport. J. Atmos. Sci., 62, 1269-1290. 